\title{
On-Line Determination of the Coordinates of a Short Circuit Point in Transmission Lines
}

\author{
Konstantin Suslov $^{1 *}$, Nafisa Solonina ${ }^{2}$, Zinaida V. Solonina ${ }^{3}$ \\ ${ }^{1-3}$ Irkutsk National Research Technical University, Russian Federation
}

\begin{abstract}
One of the challenges facing electric power systems is the failure of overhead and cable transmission lines. Short circuits pose a particular danger. This fact requires increasingly faster information acquisition and processing for estimating a system state and making preventive measures to ensure sustainable power supply with minimum loss of time. This paper is devoted to the problem of on-line determination of the coordinates of a short circuit point in the transmission line. The suggested methods are based on the assumption that the short circuit current at the transmission line beginning depends on the distance to the short circuit point.
\end{abstract}

Keywords - Phasor measurement units, short circuit point, transmission lines.

\section{INTRODUCTION}

Reduction in losses for the economic entities by the improvement in reliability and power supply quality is a topical task. At present, some measures are taken to meet the requirements on the reliability and quality of power supply. A serious problem facing electric power systems is the failure of overhead and cable transmission lines [1], [2], [3], [4]. Short circuits represent a particular danger. The probability of short circuits decreases but does not disappear with an increase in the quality of installation, reliability of insulators and conducting materials. Short circuits lead to disconnection of individual consumers and the entire regions. This imposes high requirements on the reliability and fast operation of relay protection. However, if a short circuit has occurred and relay protection has successfully operated there remains the task to promptly and where possible accurately detect the coordinates of the short circuit. This, in the end allows us to quickly restore the transmission line and place it into operation, which in turn will minimize economic losses.

There can be two types of research in the electric power industry: active experiments, and passive experiments. An active experiment can be exemplified by probing the faulty transmission line with the help of short pulses of current and assessing the response time. This time depends on the distance between the source end of the line and a short circuit point. A disadvantage of active experiments is the necessity to apply special equipment and the time to get prepared for the experiment.

A passive experiment, however, makes it possible to use modern high-speed digital technologies and obtain data (instant values of current and voltage, voltage and current phases) directly from the signals associated with the processes that occur in transmission lines after a short circuit, i. e., in real time. One of the possible solutions to this problem is related to the dependence of the currents at the source end of a line on the distance to the short circuit point [5]-[9]. However, there are reasons that decrease the accuracy of the short current point detection, namely: variations in the effective values of the voltage at the transmission line connection point, as well as the dependence of the current amplitude on the voltage phase at the time of short circuit.

\section{THE MAIN PRINCIPLES OF THE APPROACH}

We suggest two on-line methods for the determination of a short circuit point.

In the first method, the short circuit point is determined based on the time factors associated with the final velocity $\left(V_{\mathrm{F}}\right)$ of power transmission (electrical signal) along the transmission line and by the moments of response arrival at the line beginning and end.

The second method is based on the preliminary theoretical calculation of short-circuit currents at different line cutsets and on the determination of the initial effective values of short circuit currents. Here we apply the matrices of the theoretical calculations of short-circuit currents. The measured values of short-circuit currents are compared with the rated currents. The coordinates of the short circuit point are determined accurately in two stages: approximate, and accurate.

We suggest the use of phasor measurement units (PMU), upto-date digital communication tools, and a system of universe time (UT) to determine the moments of time and also information transmission to the processing center. We propose that the structural schemes of auxiliary devices which are not included in PMU should be added to the available tools.

\section{A. The First Method for the Determination of the Coordinates of a Short Circuit Point}

It is obvious that there will be a response (echo) spreading in both directions along the line, which will have the form of a front of an increasing or declining voltage or current, and the time of the response arrival at the source end or load end of the line can be recorded with a high accuracy.

We will consider the idea of the suggested method on the example of a transmission line without branches with one-way supply.

\footnotetext{
* Corresponding author.

E-mail address: souslov@istu.edu
} 


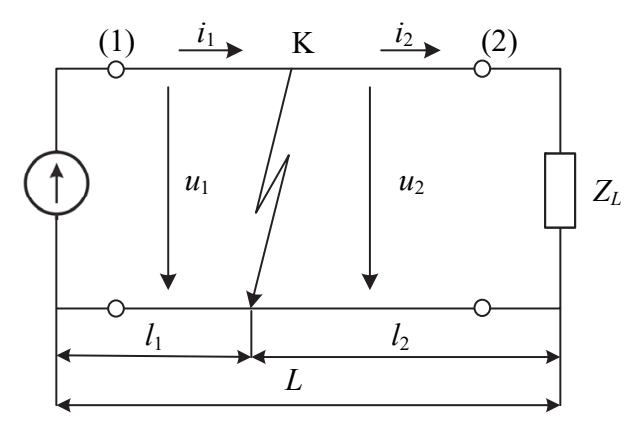

Fig. 1. Design scheme of a short circuit in a line: $L-$ length of the line; $l_{1}, l_{2}-$ distances from the short circuit point to the source and load ends of the line, respectively; $i_{1}, i_{2}, u_{1}, u_{2}-$ current and voltage of the first and second sections of the line, respectively.

Fig. 1 shows the calculation scheme for the determination of the short circuit place, taking into consideration the time of signal arrival at the source and the load ends of the line.

Let the short circuit occur at time $t_{\mathrm{sc}}$ at point $\mathrm{K}$ (Fig. 1) and a transient process start. For the sake of simplification, we make the following assumptions:

- voltage at point $\mathrm{K}$ drops to zero;

- length of the considered line is much shorter than the length of the incident current wave $\lambda(\lambda \approx 5000 \mathrm{~km}$ at the frequency of $50 \mathrm{~Hz}$ ).

We can also assume that the instant values of current and voltage in the steady state are constant along the whole line.

Fig. 2 presents a model of the line with lumped parameters for the calculation of the transient process in the line with distributed parameters.

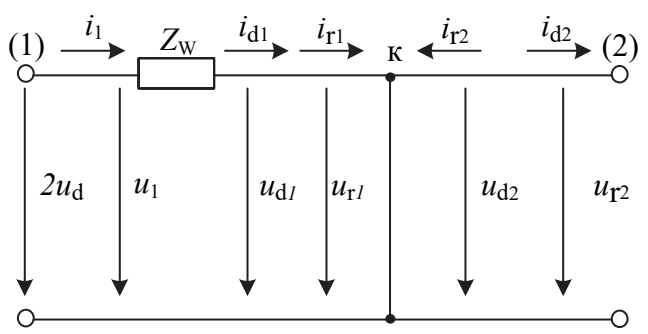

Fig. 2. Model of the line for the calculation of the transient process in the line: $Z_{\mathrm{W}}$ - wave impedance of the line; $i_{\mathrm{d} 1}$ - incident current wave of the first section, i. e., from the source end to the short circuit point; $i_{\mathrm{r} 1}-$ reflected current wave of the first section; $u_{\mathrm{d} 1}$-incident voltage wave of the first section; $u_{\mathrm{r} 1}$ - reflected voltage wave of the first section; $i_{\mathrm{d} 2}$ - incident current wave of the second section, i. e., from the short circuit point to the load end of the line; $i_{\mathrm{r} 2}$ - reflected current wave of the second section; $u_{\mathrm{d} 2}$ - incident voltage wave of the second section; $u_{\mathrm{r} 2}$ - reflected voltage wave of the second section.

It is easy to see from the model that at time $t_{\mathrm{sc}}$ :

$$
i_{\mathrm{r} 1}=i_{\mathrm{d} 1} \text { and } u_{\mathrm{r} 1}=-u_{\mathrm{d} 1} .
$$

Thus, a positive current wave front and a negative voltage wave front travel at velocity $V_{\mathrm{F}}$ in the direction from point $\mathrm{K}$ to the source end of the line (since the positive direction of the reflected current is taken opposite to the positive direction of the incident current). The fronts can be detected with the aid of the current and voltage sensors. Time $t_{1}$ of their arrival at the source end of the line can be recorded with high accuracy thanks to the clock showing the time synchronized with the universal time.

At the second section of the line, there is also a transient process. At time $t_{\mathrm{sc}}$, current $i_{2}$ and voltage $u_{2}$ at the beginning of the second section vanish, which means that

$$
\begin{gathered}
i_{\mathrm{d} 2}+i_{\mathrm{r} 2}=0, i_{\mathrm{r} 2}=-i_{\mathrm{d} 2}, \\
u_{\mathrm{d} 2}+u_{\mathrm{r} 2}=0, u_{\mathrm{r} 2}=-u_{\mathrm{d} 2} .
\end{gathered}
$$

Hence, from point $\mathrm{K}$ the negative fronts of current and voltage travel to the load end of the line. Time $t_{2}$, when these fronts arrive at the load end of the line, can be recorded with the aid of the current and voltage sensors as well as with a highprecision clock.

Let us show that knowing $t_{1}$ and $t_{2}$, we can detect the place of the short circuit. To this end we will consider a geometric model of the short-circuited transmission line. The technique of identifying the short circuit place is explained in Fig. 3.

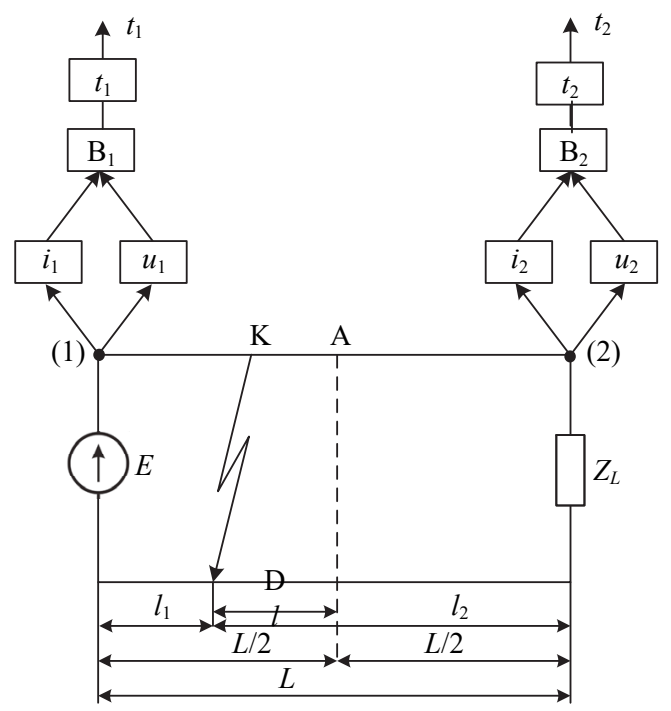

Fig. 3. A scheme of devising an algorithm for determining the place of point $\mathrm{K}$.

In Fig. 3, (1) and (2) are the sites at which the chronometers $(t)$ and the primary current $(i)$ and voltage $(u)$ sensors are installed; A is the geometric center of the line; B are the modules for processing the data from the current and voltage sensors; and $\Delta l-$ is the distance from the center of line A to the short circuit point $\mathrm{K}$.

Using this scheme, we determine $\Delta t_{1}$ - the time of wave propagation from the short circuit point to the source end of the line:

$$
\Delta t_{1}=\frac{l_{1}}{V_{\mathrm{F}}} .
$$

In the same way, we find $\Delta t_{2}$ - the time of wave propagation from the short circuit point to the load end of the line:

$$
\Delta t_{2}=\frac{l_{2}}{V_{\mathrm{F}}} \text {. }
$$

Express time $t_{1}$ of the signal (response) arrival at the source end of the line through the short circuit time:

$$
t_{1}=t_{\mathrm{sc}}+\Delta t_{1} .
$$


Similarly, find time $t_{2}$ of the response arrival at the load end of the line:

$$
t_{2}=t_{\mathrm{sc}}+\Delta t_{2}
$$

Determine the difference between the time of response arrival at the source end of the line and the time of response arrival at its load end:

$$
t_{1}-t_{2}=\Delta t_{1}-\Delta t_{2}=\frac{l_{1}}{V_{\mathrm{F}}}-\frac{l_{2}}{V_{\mathrm{F}}}=-\frac{2 \Delta l}{V_{\mathrm{F}}},
$$

and finally determine $\Delta l$ :

$$
\Delta l=-\frac{\left(t_{1}-t_{2}\right) V_{\mathrm{F}}}{2} .
$$

Knowing $\Delta l$, we find $l_{1}$ and $l_{2}$ by the equations

$$
\begin{aligned}
& l_{1}=\frac{l}{2}-\Delta l, \\
& l_{2}=\frac{l}{2}-\Delta l .
\end{aligned}
$$

If point $\mathrm{K}$ is closer to the source end of the line (to the left of point $\mathrm{A}$ ), then $\Delta l>0$. If point $\mathrm{K}$ is closer to the load end of the line, then $\Delta l<0$.

When the time aspect is taken into consideration, the issue of an accurate determination of the time instants $t_{1}$ and $t_{2}$ is particularly important.

We suggest using the available infrastructure of phasor measurement units (Fig. 4).

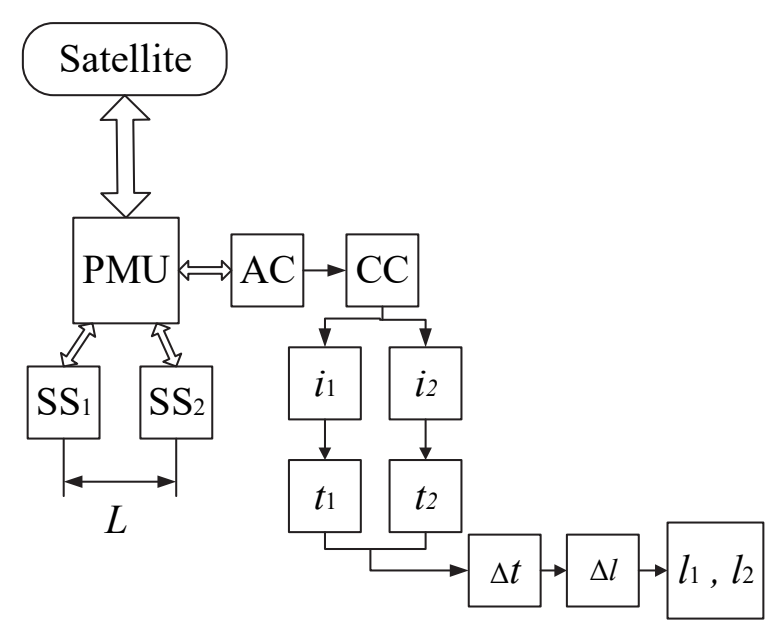

Fig. 4. PMU infrastructure, where $\mathrm{SS}_{1}$ - feeding substation; $\mathrm{SS}_{2}$ - receiving substation; $L$ - length of the line; $\mathrm{AC}$ - atomic clock; $\mathrm{CC}$ - control centre; $\mathrm{SS}_{1}$, $\mathrm{SS}_{2}$ - network substations; $t_{\mathrm{UT}}$ - time pulse of the atomic clock; $h$ - height of the satellite above the Earth in the area where the substations are located; $Q_{1}$, $Q_{2}$ - angles at which the satellite is seen from $\mathrm{SS}_{1}$ and $\mathrm{SS}_{2}$, respectively.

Phasor measurement units (PMU) make it possible to take the phasor measurements of currents and voltages at the given points of the power system. Phasor measurement implies simultaneous measurement of both the effective value and the phase of current and voltage. These parameters allow us to calculate the current values of transmitted power, voltage drops in the sections of the transmission line, power loss in the transmission line, etc. In fact, the measurement of the effective values and phases of current and voltage is not a new problem. However, such measurements, although possible, have not become widespread, since the control of the system under dynamic operating conditions requires that the data be definitely connected with the universal time.

The time measurement resolution of PMU is not sufficient to accurately determine the time of the event. Therefore, at the measurement points, we should form our own time (count) pulses with a short time interval of, for example, $10^{-9}$ seconds, using additional devices.

Consider the use of the PMU and additional devices for an accurate determination of time $t_{1}$ and time $t_{2}$. These pulses are formed by the pulse generators installed in the modules $\mathrm{B}_{1}$ and $B_{2}$, and received at the input of the pulse counters located in modules $t$. The pulses are generated with the same frequency. At the outputs of modules, we obtain $t_{1}$ and $t_{2}$, respectively, in the following form:

$$
\begin{aligned}
& t_{1}=t_{\mathrm{gps}}+n \mathrm{~d} t, \\
& t_{2}=t_{\mathrm{gps}}+m \mathrm{~d} t,
\end{aligned}
$$

where $t_{\mathrm{gps}}-\mathrm{a}$ synchronizing pulse from GPS satellite, which contains complete information about the universal time, namely: year, month, day, hour, minute, second, milliseconds;

$n, m$ - number of count pulses from the arrival of $t_{\mathrm{gps}}$ to the moment when the response to the short circuit is received at the source end and load end of the line, respectively.

We determine the difference between the time the responses arrive at the source end of the transmission line and the time they arrive at its load end, using expressions (4) and (5):

$$
t_{1}-t_{2}=(n-m) \mathrm{d} t .
$$

Knowing $t_{1}$ and $t_{2}$, we find $\Delta l, l_{1}, l_{2}$ according to expressions (1), (2), (3).

\section{B. The Second Method for the Determination of the Coordinates of a Short Circuit Point}

The main idea of the method lies in the fact that knowing circuit configuration and line parameters we can obtain an equivalent circuit for the calculation of short-circuit currents at different points of transmission line. Depending on the required accuracy, we choose a certain interval between the calculated points. The points calculated theoretically are then compared with the measured short-circuit currents.

Let us first consider the simplest case where the short-circuit current is created by one source. Fig. 1 shows a scheme of determining the location of short circuit on transmission line for the considered case.

The short-circuit currents are calculated according to the equations below:

$$
\begin{aligned}
& I_{\mathrm{sc}}^{(3)}(1)=\frac{E_{G}}{Z_{G}+Z(l)}, \\
& I_{\mathrm{sc}}^{(2)}(1)=\frac{E_{G}}{Z_{G}+2 Z(l)}, \\
& I_{\mathrm{sc}}^{(1)}(1)=\frac{E_{G}}{Z_{G}+Z(l)+Z(N)} .
\end{aligned}
$$

where $E_{\mathrm{G}}-$ system EMF (electromotive force);

$z_{G}-$ internal resistance of the system; 
$Z(N)$ - resistance from the short circuit point to the nearest ground wire;

$z(l)-$ resistance of the line section from its beginning to the short circuit location;

$I_{\mathrm{sc}}^{(3)}(l), I_{\mathrm{sc}}^{(2)}(l), I_{\mathrm{sc}}^{(1)}(l)$ - initial effective values of shortcircuit currents at three-phase, two-phase and one-phase short circuits, respectively.

\section{THE ARChITECTURE OF THE NETWORK FRAGMENT USED TO LOCATE A SHORT CIRCUIT POINT}

Thus, for each of the enumerated cases, knowing line parameters we can theoretically establish a relationship between the short-circuit currents and length $I_{\mathrm{sc}}^{(3)}(l), I_{\mathrm{sc}}^{(2)}(l)$, $I_{\mathrm{sc}}^{(1)}(l)$, and store the results in the memory in a tabular form.

There are primary current transducers at the beginning of the line in all phases of three-phase network. Their instantaneous values are processed and effective current values are calculated. At the time of short circuit, this effective value will correspond to the initial effective value of the short-circuit current. Based on the relationship between phase currents, the logical circuit determines the type of the short circuit. This makes it possible to compare real short-circuit currents with those previously calculated and thus determine the coordinate of $l$, i. e., the beginning of section on which the short circuit occurred.

Simultaneous accurate measurement of voltage at the point of connection allows the result to be adjusted considering the difference between the real voltage and the calculated one.

If to continuously measure currents and voltages (updating memory), the said values can be fixed at the moment of the short circuit. This, in particular will make it possible to determine the point of self-clearing fault. This is important since a similar short circuit can occur in the future. The identified location should be examined, and the reason for a potential short circuit should be eliminated.

Let us consider a general case where a short-circuit current on the line is considerably affected by several sources: supply system, total loads, synchronous capacitors, powerful synchronous and asynchronous motors, sources of distributed generation. The complete equivalent circuit is made up. It includes all active and passive sections of the circuit that are represented by sources of EMF and resistances according to the traditional technique for the short-circuit current calculation. After identical transformations of the complete circuit, we obtain the final equivalent circuit, which is then used to calculate short-circuit currents on line.

Fig. 5 shows an example of the final equivalent circuit with three sources.

The number of equivalent sources depends on network configuration. Therefore, the calculations should be made for different possible network configurations. From the calculation made for each circuit configuration at a rated voltage value, we obtain a matrix of current values for the different types of short circuit at the specified values of $l$. An example of the matrix is given in Table I.

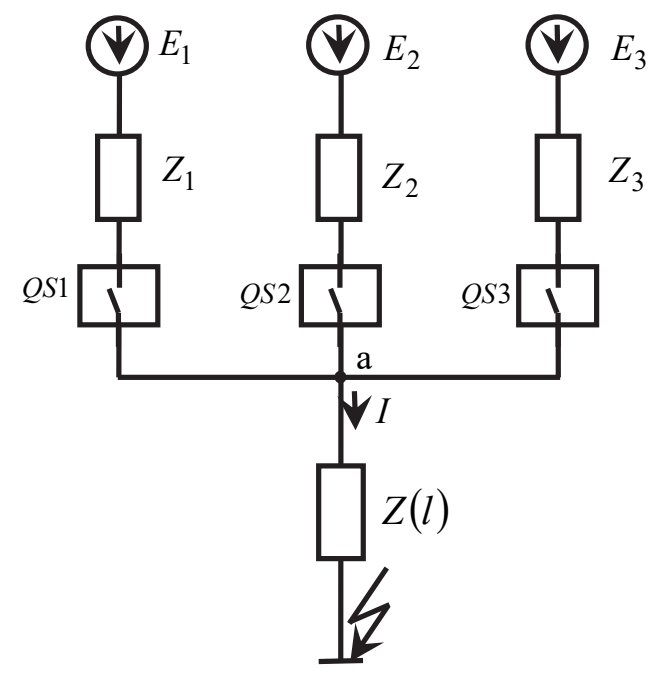

Fig. 5. Final equivalent circuit, where $E_{1}, E_{2}, E_{3}-$ sources that have a considerable impact on the short-circuit current of line; $Z_{1}, Z_{2}, Z_{3}$ - equivalent resistances of a circuit section from sources to the beginning of the considered line; $Q S 1, Q S 2, Q S 3$ - circuit breakers; $Z(l)$ - resistance of the line section from its beginning to the short circuit point located at a distance $l$ from the beginning of the line.

TABLE I

An EXAMPLE of Data Matrix For the First Stage of FAULt Location DETECTION

\begin{tabular}{|l|l|l|l|l|}
\hline $\boldsymbol{i}$ & $l_{i}=i \Delta l, \mathrm{~m}$ & $I_{i}^{(3)}, \mathrm{kA}$ & $I_{i}^{(2)}, \mathrm{kA}$ & $I_{i}^{(1)}, \mathrm{kA}$ \\
\hline 1 & $l_{1}=\Delta l$ & $I_{1}^{(3)}$ & $I_{1}^{(2)}$ & $I_{1}^{(1)}$ \\
\hline 2 & $l_{2}=2 \Delta l$ & $I_{2}^{(3)}$ & $I_{2}^{(2)}$ & $I_{2}^{(1)}$ \\
\hline$\ldots$ & $\ldots$ & $\ldots$ & $\ldots$ & $\ldots$ \\
\hline$i-1$ & $l_{i-1}=(i-1) \Delta l$ & $I_{i-1}^{(3)}$ & $I_{i-1}^{(2)}$ & $I_{i-1}^{(1)}$ \\
\hline$i$ & $l_{i}=i \Delta l$ & $I_{i}^{(3)}$ & $I_{i}^{(2)}$ & $I_{i}^{(1)}$ \\
\hline$i+1$ & $l_{i+1}=(i+1) \Delta l$ & $I_{i+1}^{(3)}$ & $I_{i+1}^{(2)}$ & $I_{i+1}^{(1)}$ \\
\hline$\ldots$ & $\ldots$ & $\ldots$ & $\ldots$ & $\ldots$ \\
\hline$n$ & $l_{n}=n \Delta l$ & $I_{n}^{(3)}$ & $I_{n}^{(2)}$ & $I_{n}^{(1)}$ \\
\hline
\end{tabular}

where $i$ - number of calculated points of a possible short circuit on the interval $\Delta l ; l_{i}$ - distance from the beginning of a line to the fault location; $n$-total number of points to be calculated; $\Delta l$-sampling step (in meters).

It is supposed that there are primary transducers of current and voltage at the beginning of the line and that effective values of current and voltage are continuously measured. The indicated values are stored in the data concentrator and constantly updated. The location of a line fault is detected in two stages: preliminary, and final.

The algorithm for preliminary detection of the coordinates of the short-circuit current location is as follows. Knowing the short-circuit currents of different phases, we determine the type of the short circuit, namely: if the currents of three phases are 
almost equal, this is a three-phase symmetrical short circuit; if two currents are equal and considerably exceed the third current, this is a two-phase asymmetrical short circuit; if the current of one of the phases considerably exceeds the currents of other phases, this is a one-phase short circuit. Knowing the value of the voltage at point "a" (Fig. 5) at the time instant preceding the short circuit, we adjust the data of the matrix, supposing that the calculated currents are linearly related to the voltage. Knowing the type of the short circuit, we find an interval from the matrix, within which the measured initial effective value of the short-circuit current lies. Let it be established that the short circuit is a three-phase one. Determine an interval that meets the following inequality:

$$
I_{\mathrm{sc}(i+1)}^{(3)}<I_{\mathrm{sc}}^{(3)^{*}}<I_{\mathrm{sc}(i)}^{(3)}
$$

where $I_{\mathrm{sc}(i+1)}^{(3)}, I_{\mathrm{sc}(i)}^{(3)}-$ calculated values of short-circuit current at calculated points $(i+1)$ and $(i)$, respectively; $I_{\mathrm{sc}}^{(3)^{*}}-$ value of short-circuit current obtained from the measurement. We suppose that $I_{\mathrm{sc}}^{(3)^{*}} \approx I_{\mathrm{sc}(i)}^{(3)}$ and determine an interval from $(i-1)$ to $(i+1)$, i.e., $l=l_{i}$, within which the short circuit occurred.

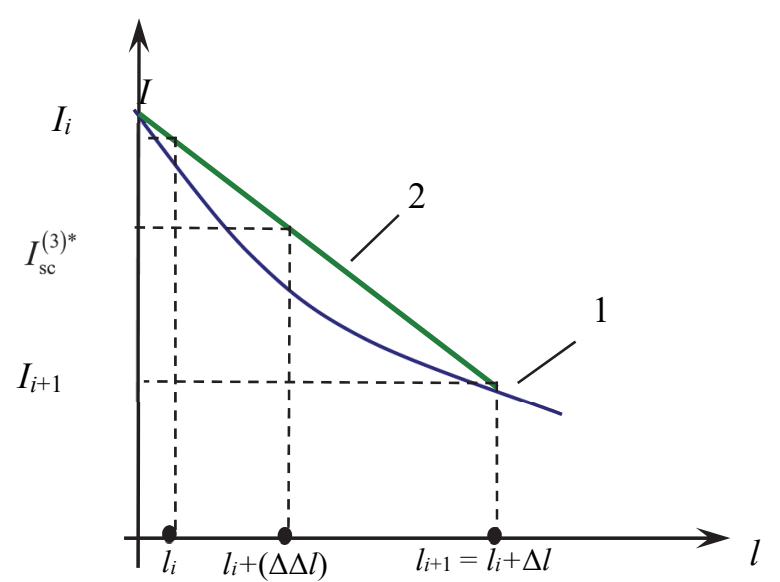

Fig. 6. The second stage of determining the place of a short circuit (1 theoretical dependence of $I(l) ; 2$ - linear approximation of $I(l)) ; l_{i}, l_{(i+1)}$ beginning and end of the interval at the first stage of determining the place of a short circuit; $\Delta \Delta l$ - interval of the second stage of determing; $I_{\mathrm{sc}}-$ value of the short-circuit current).

To accurately determine the coordinates of the short circuit, the dependence of $I(l)$ (curve 1), the interval is replaced by a straight line 2 (Fig. 6).

The linear dependence can be provided by using Rogowski coil instead of current transformers. In essence, the former is a current-to-voltage transducer. This is convenient for further conversion of information from the analog form into the digital one.

The high accuracy of current measurement is due to the absence of the magnetic core in it. Since there is no ferromagnetic coil in Rogowski coil, the output signal (voltage) will linearly depend on the input (measured) value (current).

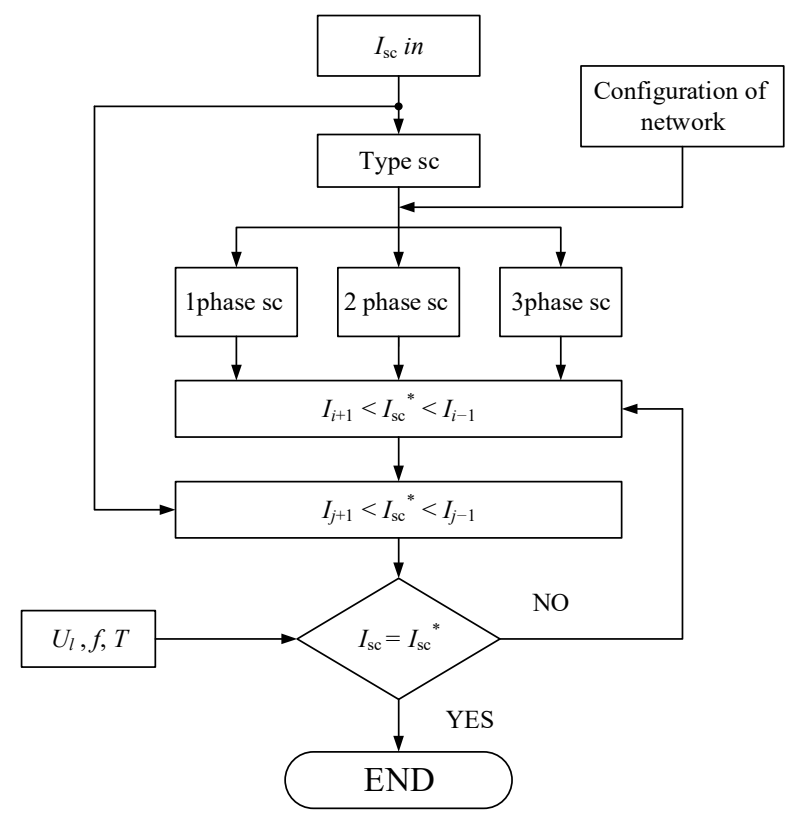

Fig. 7. The algorithm for the on-line determination of a short circuit point in the transmission line.

The effective values of linear voltages $U$ at the point of line connection to the system are measured. The current value of frequency $f$ and period $T$ of voltage are determined. These data are delivered from the primary detectors and continuously updated keeping in memory their values which are received directly before a short circuit. The accurate value of the period is necessary for determining the initial value of the periodic component of short-circuit current. The procedure is as follows. At time intervals multiple of the accurate value of the period, the instantaneous current values are determined and fixed; the running value of the effective current value is determined; and the period with the highest effective running value is determined. The array of the numerical values of the determined period is directed for further processing, namely, the methods of digital filtering separate only the fundamental harmonic and determine its effective value which is compared subsequently with the theoretically determined values.

The initial effective values of short-circuit currents of all three phases are measured during the first period after a short circuit. The type of the short circuit - single-, double-, or threephase - is determined according to expressions (6)-(8). The logical unit determines a network configuration which is changed by using circuit breakers. Therefore, the state of network configuration at the moment can be determined by the state of circuit breakers. The information about the state of circuit breakers is transmitted by the supervisory control channels. The network configuration can be specified directly by the dispatcher. The logical unit of the first stage of determining the section, within which the short circuit occurred, operates as follows. If the short circuit type is known, then from the array of calculated currents of the transmission line for a specific configuration (Table 1) we determine an interval within which the measured initial effective value of the short-circuit current lies. Then the information is transmitted to the logical unit of the second stage of determining the coordinates of the 
short circuit location. The information from the unit is compared with the rated value. If they are equal, the coordinates of the short circuit location will be exact.

The proposed method is very promising, since it mainly uses PMU devices and the costs related to the development and use of additional devices are insufficient.

This method allows the coordinates of the damage in real time to be determined. The error is not more than 50 meters. This figure will be adjusted to the experiments conducted.

However, it is needed to pay attention to the following: when calculating the initial effective value of the short-circuits current, it is assumed that the voltage and, consequently, the line current are sinusoidal. In the actual conditions, the currents and voltages in transmission lines are non-sinusoidal, i. e., contain high harmonics [10]. Therefore, the high harmonics can significantly increase the initial effective value of the measured current compared with the calculated one. This causes a corresponding error in determining the coordinates of a short circuit.

For example, if a cable length is $100 \mathrm{~km}$, the error of $1 \%$ will seriously complicate the search for the short circuit location of the transmission line. To reduce this component of the error, it is necessary to select the fundamental harmonic output voltage Rogowski coil.

It is necessary to memorize the instantaneous value of the short circuit current during the first period immediately after short circuit. The logical unit processes the data and selects the initial value of the fundamental harmonic, which is compared with the calculated tabulated values.

Thus, as distinct from the known methods, the application of information technologies and Rogowski coil makes it possible to determine the precise coordinates of the short circuit location on-line with the required accuracy.

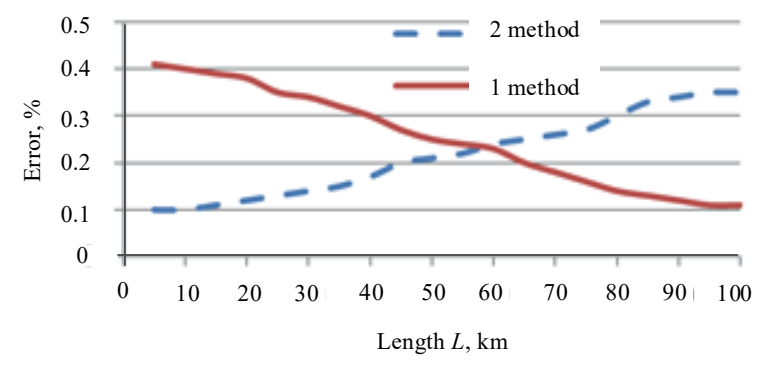

Fig. 9. Comparison of the accuracy of determining the fault location.

The estimation error in determining the fault location by two methods depending on the line length has been investigated. The results are shown in Fig. 9. As a result of the comparison, it is obvious that the second method is more accurate in the case when the length of the line is small. When the length of the line exceeds $50 \mathrm{~km}$, the first method should be used.

\section{CONCLUSION}

Two methods are suggested for the on-line determination of the short circuit point in transmission lines. In the first method, the short circuit point is determined based on the application of time factors associated with the final velocity of power transmission (electrical signal) along the transmission line and by the moments of response arrival at the line beginning and end.

The second method is based on the preliminary theoretical calculation of short-circuit currents at different line cutsets and on the determination of the initial effective values of shortcircuit currents. The method applies the matrices of the theoretical calculations of short-circuit currents. The measured values of short-circuit currents are compared with the rated currents. The coordinates of the short circuit location are exactly determined at two stages: approximate, and accurate.

It is suggested that the moments of time and information transmission to the processing center should be determined with the help of PMU, up-to-date tools of digital communication, and UT system.

We propose that the structural schemes of auxiliary devices, which are not foreseen in PMU, should be added to the available tools.

\section{REFERENCES}

[1] Z. Yang, H. Zhong, Q. Xia, and C. Kang, "Optimal transmission switching with short-circuit current limitation constraints," in 2016 IEEE Power and Energy Society General Meeting (PESGM), Jul. 2016. https://doi.org/10.1109/pesgm.2016.7741808

[2] Y. Chai, C. Jiang, K. Zhang, and S. Xu, "Safe operation improvement of an electrical power system by superconducting fault current limiters," ir 2016 Chinese Control and Decision Conference (CCDC), May 2016. https://doi.org/10.1109/ccdc.2016.7531187

[3] F. Gao, S. Ai, R. Ding, and Y. Huang, "Development and experiment of fast breaker-type fault current limiter," in 2015 5th International Conference on Electric Utility Deregulation and Restructuring and Power Technologies (DRPT), Nov. 2015. pp. 1790-1794. https://doi.org/10.1109/drpt.2015.7432533

[4] Z. Wu, L. T. Zora, and A. G. Phadke, "Simultaneous transmission line parameter and PMU measurement calibration," in 2015 IEEE Power \& Energy Society General Meeting, Jul. 2015. https://doi.org/10.1109/pesgm.2015.7286115

[5] M. Gilany, E. S. Tag El Din, M. M. Abdel Aziz, and D. Khalil Ibrahim, "An accurate scheme for fault location in combined overhead line with underground power cable," IEEE Power Engineering Society General Meeting, 2005. https://doi.org/10.1109/pes.2005.1489308

[6] A. A. Hajjar, M. M. Mansour and H. A. Talaat, "High-phase order power transmission lines relaying approach based on the wavelet analysis of the fault generated traveling waves," in 39th International Universities Power Engineering Conference, 2004. UPEC 2004, Bristol, UK, 2004, pp. 805809 vol. 1.

[7] G. D. Ferreira, D. S. Gazzana, A. S. Bretas, and A. S. Netto, “A unified impedance-based fault location method for generalized distribution systems," in 2012 IEEE Power and Energy Society General Meeting, Jul. 2012. https://doi.org/10.1109/pesgm.2012.6345512

[8] C. Wang, B. Zhang, G. Li, L. Yan, and J. Zhao, "One practical method of fault location for transmission lines based on dual-voltage fault components," in 2011 International Conference on Electrical and Control Engineering, Sep. 2011. https://doi.org/10.1109/iceceng.2011.6057787

[9] F. T. J. Van Erp, F. Provoost, A. P. J. Van Deursen, and P. L. J. Hesen, "Modelling and live measurements of step and touch voltages at LV customers in urban areas caused by MV faults," in 18th International Conference and Exhibition on Electricity Distribution (CIRED 2005), 2005. https://doi.org/10.1049/cp:20051001

[10] J. Arrillaga and N. R. Watson, Power system harmonics, Chichester: Wiley, 2nd ed., 2003. 